\title{
Research on Method of Evaluating Anti-wave Ability of Deepwater Long-line Rope Aquaculture Facility
}

\author{
Ya ZHANG ${ }^{1, a}$, Jian LUAN ${ }^{1, b}$, Guang-Fa ZHANG ${ }^{1, c, *}$, Jun LIANG ${ }^{2, c}$, \\ Yong $\mathrm{CHEN}^{1, d}$ \\ ${ }^{1}$ Dalian Ocean University, Dalian 116023, China \\ ${ }^{2}$ Zhangzidao Group CO. LTD, Dalian 116001, China \\ azhangya@dlou.edu.cn, bluajian@dlou.eud.cn, czhangguangfa@dlou.edu.cn, \\ dchenyong@dlou.edu.cn \\ ${ }^{*}$ Corresponding author
}

Keywords: Deepwater Long-line Aquaculture Facility, Anti-wave Ability, Hydrodynamic.

\begin{abstract}
Based on statics assumption and simplifications, the force and failure mode of deepwater long-line rope aquaculture facility system were analyzed, and maximum tensions of the mooring rope in the system in different sea wave heights $(1 \sim 7 \mathrm{~m})$ were calculated to compared to its break strength and mooring force of anchoring pile for evaluating anti-wave ability of the aquaculture facility. Through this method, the model of evaluating anti-wave ability of deepwater long-line rope aquaculture facility was established. As an instance, the anti-wave abilities of two scales of deepwater long-line rope aquaculture facilities erected in 30 meters deep sea waters of Zhangzidao culture sea area were analyzed by using the evaluation method and model. The results of the theory analysis show that the anti-wave abilities of the two scales $(300 \mathrm{~m}$ and $200 \mathrm{~m}$ main rope length) of aquaculture facilities system were respectively 5 6 level of sea wave (7 8 Beaufort wind grade) and 6 7 level of sea wave (8 9 Beaufort wind grade) in $1.5 \mathrm{~m} / \mathrm{s}$ sea current. By comparison, the value of calculation is good to worth with actual measurement. Thus the method and model of evaluating anti-wave ability of deepwater long-line rope aquaculture facility could be referenced for designing the scales of deepwater long-line rope aquaculture facility in the preliminary design stage.
\end{abstract}

\section{Introduction}

Aquaculture raft originated from Japan, which is widely used in oysters, scallops and large algae culture. From 1950s, Chinese seaweed aquaculture technology began to improve and mature, and has already developed into a multi-species aquaculture technology involving algae, shellfish, abalone, sea cucumber, sea urchins, crabs and other species, the scale of which is growing and gradually expanding to deepwater. Deepwater long-line aquaculture facility belongs to a raft culture method, the structure of which is mainly constituted by buoys, net cages, main ropes, mooring ropes and the anchor stakes. The facility stays in deep-open waters with winds, waves, urgent flow, and is affected by complex sea conditions; the safety and reliability of its structure will directly affect the results of the entire aquaculture production. Therefore it's needed to take the culture process and the influence of ocean winds, waves, and flows into consideration during its structural design, and doing structural design based on studies and analysis of the force, motion characteristics and wind resistance.

Some researchers studied the raft aquaculture structure by using finite element method or lumped-mass method. Grant J[1] simulated an aquaculture raft structure with finite element method, E. Lienand A. Fredheim[2] did comparison study for the anti-wave abilities of long-line and floating tube raft culture systems with commercial software. In recent years, many studies about coastal aquaculture facilities were carried out in China, including aquaculture regional planning, structural dynamic analysis, the impact of waves and currents on the structure, aquaculture facilities anti-wave abilities, structural design and other aspects. Wangjing Kun[3] used Pro/Engineer and Ansys software as a platform for modeling and analysis, and carried out researches on parametric design for structures of aquaculture facilities with virtual design technology studies. Li Guanying[4] used physical experiments and numerical models methods to study the dynamic characteristics of deepwater oyster culture facilities. Deng Tui $[5,6]$ simulated and analyzed the overall movement of a 
new aquaculture facility system under sea waves by using lumped mass method. Cui Yong[7] analyzed the maximum displacement of buoys and net cages of aquaculture facilities as well as the stress condition and anti-wave abilities of the mooring rope with finite element method. Currently, researches on aquaculture facility and its related area in China mainly focus on coastal waters within 20 meters deep, offshore deepwater aquaculture technology still in its infancy, especially for deepwater long-line aquaculture facility, there are few studies of which both at home and abroad.

Under statics simplified conditions, the stress and structure of deepwater long-line rope aquaculture facility (hereinafter referred to as aquaculture facility) were analyzed, and maximum tensions of the mooring rope in the system in different sea wave heights $(1 \sim 7 \mathrm{~m})$ were calculated to compared to its break strength and mooring force of anchoring pile for evaluating anti-wave ability of the aquaculture facility. Through this method, the model of evaluating anti-wave ability of aquaculture facilities was established. As an instance, the anti-wave abilities of two scales $(300 \mathrm{~m}$ and $200 \mathrm{~m}$ main rope length) of aquaculture facilities erected in 30 meters deep sea waters of Zhangzidao culture sea area were analyzed by using the evaluation method and model. By comparison, the value of calculation is good to worth with actual measurement. Thus the method and model of evaluating anti-wave ability of aquaculture facility could be referenced for designing the scales of aquaculture facility in the preliminary design stage.

\section{The Structural Style of Deepwater Long-line Rope Aquaculture Facility}

The structure of deepwater long-line rope aquaculture facility (hereinafter referred to as the raft system) as shown in Figure 1, is composed mainly of buoys, a main rope, some aquaculture net cages, two sets of mooring ropes and anchor stakes. The raft system is suspended in sea water by the buoys connected to main rope by a lifting line to, and anchored by the stake through mooring ropes which connected with main rope at two ends. The net cages are suspended on main rope to implement aquaculture. The length of main rope is $200 \sim 400 \mathrm{~m}$, and the diameter of a buoy is $35 \sim 45 \mathrm{~cm}$. The aquaculture cage is a cylinder structure with diameter $30 \sim 40 \mathrm{~cm}$ and length $2 \sim 5 \mathrm{~m}$. The anchor angle between mooring rope and seafloor is generally 10 degrees, and water depth $30 \sim 40 \mathrm{~m}$, so the length of mooring rope and connecting point with main rope can be calculated by mooring line theory.

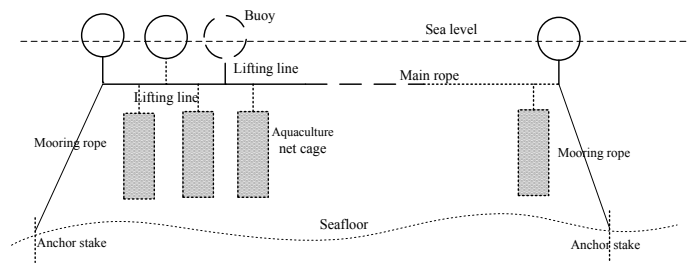

Fig. 1 Structure Diagram of Deepwater Long-line Rope Aquaculture Facility

The buoyancy of raft system is provided by buoys and the structure stability is ensured by the anchorage force from anchor stake sunk into seafloor. The raft system will move and deform in the environmental load and the tension forces will be produced inner the rope and between mooring rope and anchor stake that will lead to rope broken off or anchor stake broken away from seafloor and cause to severe damages. So the raft system has three failure modes as follows:

(1) The mooring rope is broken due to the maximum tension force beyond its strength.

(2) The anchor stake is broken away from seafloor due to the maximum tension force beyond the stake's grasping force with seafloor.

(3) The anchor stake is broken away from seafloor due to the mooring rope fatigue[4].

For the third failure mode of the raft system, the dynamic analysis of the raft system and further mooring rope fatigue analysis should be carried on. This paper will study the anti-wave ability of the raft system based on the first two failure modes.

\section{Estimation Method of Anti-wave Ability of Aquaculture Facility}

The movement, stress and damage situation of aquaculture facility under the action sea wind, wave and current are very complex, as well as the interaction between system components. Water depth, the scale of sea wind and waves, and the current size are the main factors that influence the stability 
and anti-wave ability of aquaculture facility. Anti-wave ability of aquaculture facility depends on the system reserve buoyancy, wave strength, the fracture strength of all ropes in the system, the grip of anchor stake, etc. It is very difficult to carry on the theoretical analysis on its anti-wave ability. Through the analysis of failure modes of aquaculture facility in section 1, it can be seen that its failure is mostly due to the maximum tension of mooring ropes exceeding the material ultimate strength or the grip of anchor stake. Therefore, through certain hypothesis and simplification, based on the principle of statics, the maximum tension of mooring ropes of the aquaculture facility in different sea wave levels can be calculated, which is compared with the material ultimate strength and the grip of anchor stake, then to get the maximum sea wave level the facility can resist, and according to which, preliminary evaluation can be made on anti-wave ability of aquaculture facility. In the calculation, forces in horizontal and vertical directions are firstly calculated the in certain sea conditions, which are acting on the buoys, net cages, main ropes and mooring ropes of aquaculture facility system, and then merge them, so as to get the maximum force of the mooring rope.

In the case that it doesn't have a greater impact on the calculation results, this paper makes the following static assumption and simplification:

(1) Ignore the environmental wind pressure of the system for most components of the aquaculture facility will sink below sea level when large winds and waves.

(2) Ignore actual wearing of all ropes and check the fracture strength as new material.

\section{Hydrodynamic Calculation of Aquaculture Facility System}

\section{Hydrodynamic Models}

The components in raft system (buoy, cage, rope) belong to small scale marine structures on which the fluent loads could be calculated by the Morison method ${ }^{[8]}$. According to Morison method, if the diameter of marine structure is small compared to wavelength ( $D / L \leq 0.15)$, the sea wave field will transmit without influence of the marine structure. So the fluent load on the structure will be composed two forces, one is drag force produced by the undisturbed wave speed field, and the other is inertial force produced by the wave acceleration field. Thus fluent load on the structure can be represented as follows:

$$
\begin{aligned}
& f=f_{d}+f_{m} \\
& f_{d}=C_{d} \rho A_{d}|u| u \\
& f_{m}=C_{m} \rho A_{m} a
\end{aligned}
$$

Where $C_{d}$ is drag force coefficient, $C_{m}$ is inertial force coefficient. $A_{d}, A_{m}$ is projected area of the structure to flow direction for calculating drag force and inertial force respectively. $C_{d}, C_{m}, A_{d}$ and $A_{m}$ can be selected on the type of the structure according to the figure9.3 and figure9.4 in the 266th page of the reference[8]. $\mathrm{u}$ and a is the speed and acceleration of water particle respectively which can be gotten from the selected wave theory model.

For the buoy in the raft system, as ball structure, the above parameters selected as $C_{d}=0.5, C_{m}=1.5$, $A_{d}=\pi D^{2} / 4, A_{m}=\pi D^{3} / 6$, where is diameter of the buoy.

For net cages, simplified as cylinder with certain permeability, and the fluent force can be calculated as eq(4):

$$
f_{c}=k f
$$

Where $\mathrm{f}$ is the wave force subjected to cage cylinder calculated according to the above Morison equations (eq(1) (3)) in which $C_{d}=1.0, C_{m}=2.0, A_{d}=0.5 D d l, A_{m}=0.25 \pi D^{2} d l, \mathrm{D}$ is the diameter of the cylinder, $\mathrm{dl}$ is cylinder unit length. Integrating the cylindrical net cage along the cylinder length direction ( $\mathrm{z}$ direction), the wave force of the whole cylinder can be got. $\mathrm{k}$ is a coefficient considering 
cage permeability which related to mesh size and marine biofouling of the net cage. For the cage with aquaculture products filling the whole net cage space and plentiful marine biofouling, it can be set the permeability coefficient $k=1$ to calculate the maximum fluent load.

The fluid load on ropes (main ropes and mooring ropes) can be calculated with the tilt cylinder hydrodynamic calculation method[9].

\section{Current Class Definitions and Wave Model Selection}

In the calculation, the wave scales are defined by reference [10], aquaculture facility is calculated under sea conditions of different wave heights from 3 to 8 waves (a total of 7 scales) in this paper. When calculating the hydrodynamic of each component by using Morison method, firstly calculate the velocity and acceleration of water particles by choosing the appropriate wave model, then superimposed with which of the waters, so to get the velocity and acceleration of water particles under a certain sea condition.

In order to calculate different grades of wave, the large wave height should be considered and the nonlinear wave force cannot be ignored, thereby the two order Stokes wave theory is employed to calculate the speed $u$ and acceleration a of water particle as following eq(5) (8)[11].

$$
\begin{aligned}
& u_{x}=\frac{\pi H}{T} \frac{\operatorname{ch}(k(z+d))}{s h(k d)} \cos (k x-w t)+\frac{3}{4} \frac{\pi H}{T} \frac{\pi H}{L} \frac{\operatorname{ch}(2 k(z+d))}{s^{4}(k d)} \cos ^{2}(k x-w t) \\
& u_{z}=\frac{\pi H}{T} \frac{\operatorname{sh}(k(z+d))}{s h(k d)} \sin (k x-w t)+\frac{3}{4} \frac{\pi H}{T} \frac{\pi H}{L} \frac{\operatorname{sh}(2 k(z+d))}{s^{4}(k d)} \sin ^{2}(k x-w t) \\
& a_{x}=2 \frac{\pi^{2} H}{T^{2}} \frac{\operatorname{ch}(k(z+d))}{s h(k d)} \sin (k x-w t)+3 \frac{\pi^{2} H}{T^{2}} \frac{\pi H}{L} \frac{\operatorname{ch}(2 k(z+d))}{s^{4}(k d)} \sin ^{2}(k x-w t) \\
& a_{z}=-2 \frac{\pi^{2} H}{T^{2}} \frac{\operatorname{sh}(k(z+d))}{s h(k d)} \cos (k x-w t)-3 \frac{\pi^{2} H}{T^{2}} \frac{\pi H}{L} \frac{\operatorname{sh}(2 k(z+d))}{s h^{4}(k d)} \cos ^{2}(k x-w t)
\end{aligned}
$$

Where $\mathrm{H}$ is wave height, $\mathrm{T}$ is wave period, which can be input by the wave level, $\omega$ is wave angular frequency, $\omega=2 \pi / T, \mathrm{k}$ is wave number which calculated by the wave dispersion relation equation $k=\frac{\omega^{2}}{g} t h^{-1}(k d), \mathrm{L}$ is wave length, $L=2 \pi / k, \mathrm{~d}$ is environment water depth, $z+d$ is water depth of calculated point.

\section{Examples of Calculation and Analysis}

Using the above methods, the anti-wave abilities of two scales aquaculture facilities erected in 30 meters deep sea waters of Zhangzidao culture sea area were estimated, the length of the main rope of the two facilities were respectively $300 \mathrm{~m}$ and $200 \mathrm{~m}$. Aquaculture facility erected in 30 meters deep sea waters of Zhangzidao are a typical culture device, the length of the main rope of which is between $200 \mathrm{~m}$ and $300 \mathrm{~m}$, the diameter of a buoy is $0.42 \mathrm{~m}$, the number of buoys is from 40 to 80 , actual measurement shows that the maximum grip of anchor stake is $110 \mathrm{kN}$ to $150 \mathrm{kN}$, the flow velocity is $1 \mathrm{~m} / \mathrm{s}$ to $1.5 \mathrm{~m} / \mathrm{s}$. Main ropes and mooring ropes are made of polypropylene material, with a diameter between $20 \mathrm{~cm}$ to $40 \mathrm{~cm}$, the fracture strength of two strands of twisted polypropylene mooring ropes is $116 \mathrm{kN}$ to $250 \mathrm{kN}$.

\section{Analysis of Anti-wave Abilities of Two Aquaculture Facilities}

By calculation, maximum tensions of two types of aquaculture facilities in different wave scales can be got, Figure 2 shows the maximum tensions trend changing with wave heights, according to the figure, in the waters of a flow velocity $1.5 \mathrm{~m} / \mathrm{s}$, the anti-wave abilities of two types of aquaculture facilities with the main rope length of $300 \mathrm{~m}$ and $200 \mathrm{~m}$ is respectively 5 to 6 wave scale (corresponding 7 to 8 wind scale) and 6 to 7 wave scale (corresponding 8 to 9 wind scale). 


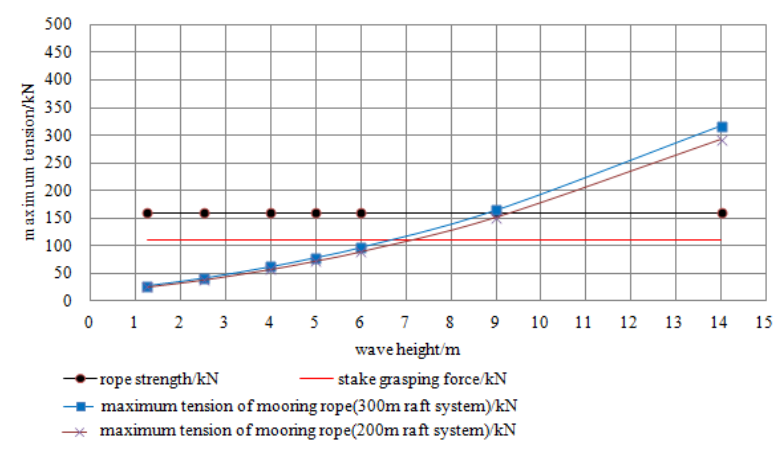

Fig. 2 Two Types of Aquaculture Facilities' Maximum Tensions Trend Chart with Wave Height Changing

\section{Effects of Aquaculture Facilities' Structure Scale on Anti-wave Abilities}

The structure scale of aquaculture facility mainly refers to the main rope length, the number of net cages and the number of buoys, the anti-wave abilities of aquaculture facilities with the main rope length from $200 \mathrm{~m}$ to $300 \mathrm{~m}$ were calculated, the results shown in Table 1 . The calculation results show that the larger scale the aquaculture facility was, the bigger maximum tension of mooring rope was, and the poorer its anti-wave ability was; but the smaller-scale aquaculture facility would affect the economic benefits of culture. Thus in the choice of structure scale, it should be a larger scale, under the premise of its anti-wave ability.

Tab. 1 Anti-Wave Ability of Different Scale of Aquaculture Facilities

\begin{tabular}{ccccccc}
\hline $\begin{array}{c}\text { Main rope length/number } \\
\text { of buoy, cage }\end{array}$ & $300 \mathrm{~m} / 60,150$ & $\begin{array}{c}280 \mathrm{~m} / 56, \\
140\end{array}$ & $260 \mathrm{~m} / 52,130$ & $240 \mathrm{~m} / 48,120$ & $\begin{array}{c}220 \mathrm{~m} / 43, \\
110\end{array}$ & $\begin{array}{c}200 \mathrm{~m} / 40, \\
100\end{array}$ \\
\hline $\begin{array}{c}\text { Anti-wave grade/wave } \\
\text { height }\end{array}$ & $6 / 5.0 \mathrm{~m}$ & $6 / 5.3 \mathrm{~m}$ & $6 / 5.8 \mathrm{~m}$ & $6 / 6.1 \mathrm{~m}$ & $7 / 6.7 \mathrm{~m}$ & $>7 / 7.2 \mathrm{~m}$ \\
\hline
\end{tabular}

\section{Effects of Current Directions on Anti-wave Ability}

By calculation, when the current incidence angle changes from $0^{\circ}$ to $90^{\circ}$, the maximum tension of mooring rope gradually become smaller, anti-wave ability become better. The reason is that when the current incident angle is $0^{\circ}$, all tension of the structure will be borne by the mooring rope at only one end, the tension is maximum at this moment; when the angle increases, mooring ropes at both ends bear the structural hydrodynamic together, therefore the maximum tension one single mooring rope will gradually decrease, and will reach a minimum value at $90^{\circ}$. Thus, the anti-wave ability of aquaculture facility is worst when the incident angle is $0^{\circ}$. When making arrangements for aquaculture facilities, the direction of which should avoid the direction consistent with the current.

\section{Experimental Verification}

Tab. 2 Comparison of Calculating Value to Actual Measurement on Mooring Rope'S Maximum Tension

\begin{tabular}{rcccccc}
\hline $\begin{array}{l}\text { Wind } \\
\text { grade }\end{array}$ & $\begin{array}{c}\text { Actual wave } \\
\text { height }(\mathrm{m})\end{array}$ & $\begin{array}{c}\text { Wave height for } \\
\text { Calculation }(\mathrm{m})\end{array}$ & $\begin{array}{c}\text { flow velocity } \\
(\mathrm{m} / \mathrm{s})\end{array}$ & $\begin{array}{c}\text { Calculated } \\
\text { maximum tension } \\
(\mathrm{kN})\end{array}$ & $\begin{array}{c}\text { Measured } \\
\text { maximum tension } \\
(\mathrm{kN})\end{array}$ & $\begin{array}{c}\text { Relative error } \\
(\%)\end{array}$ \\
\hline 6 & $3.5 \sim 4.1$ & 4.0 & 1.22 & 62.5 & 57.4 & $8.8 \%$ \\
7 & $4.0 \sim 4.6$ & 5.0 & 1.18 & 78.8 & 72.7 & $8.4 \%$ \\
8 & $5.2 \sim 6.2$ & 6.0 & 1.31 & 97.2 & 90.6 & $7.3 \%$ \\
\hline
\end{tabular}

In order to verify the theoretical calculation results, the maximum tension of mooring ropes in a aquaculture facility with the main rope length $300 \mathrm{~m}$ was measured under three sea conditions $(6,7,8$ wind scales) in certain sea area. The tension of mooring ropes at both ends of the aquaculture facility was measured by waterproof dynamometer with $50 \mathrm{t}$ range, wind scales and wave heights were from local marine observation data, the current velocity was measured by current meter. Actual measurements are shown in Table 2. The actual values and the calculated values are compared in Table 2, from where it can be seen that the calculated values agree well with the actual measurements. In addition, the company's culture accident reports show that, in several pushing-raft accidents, the 
structural failure of aquaculture facility with the main rope length $300 \mathrm{~m}$ is more than that of $200 \mathrm{~m}$. Most of the pushing-raft accidents happen due to the slipping of anchor stake under 8, 9 wind scale conditions. This is consistent with the results of the above analysis and calculation.

\section{Conclusions}

Through static assumption and simplification on aquaculture facilities, according to the stress characteristic, the model for evaluating anti-wave ability of deepwater long-line rope aquaculture facility can be established, based on which qualitative conclusions can be made on aquaculture facility's anti-wave ability, and then it could be referenced for designing structure scales of aquaculture facilities in the preliminary design stage. Instance case shows that the larger scale aquaculture facility structure will have a bigger maximum tension of mooring rope, thus it will have a less anti-wave ability. Moreover the anti-wave ability of aquaculture facility will reduce with the wave incident angle (namely the layout angle of the aquaculture facility) changing small. In order to improve the anti-wave ability of aquaculture facility, the structure scale and layout angle (current direction) should be taken into consideration when designing or remaking deepwater long-line rope aquaculture facility.

\section{Acknowledgments}

The authors acknowledge the supports of the National Science and Technology Planning Project of China (2012BAD18B0), and Horizontal Subject ("Optimal design of scallop aquaculture facility") from Dalian Zhangzidao Group CO. LTD, and express profound thanks for their financial support.

\section{References}

[1] Grant J, Bacher C. A numerical model of flow modification induced by suspended aquaculture in a Chinese bay[J]. Canadian Journal of Fisheries and Aquatic Sciences, 2001, 58:1003-1011.

[2]E. Lien, A. Fredheim. Development of Long tube Mussel Systems for Cultivation of Blue Mussels(Mytilis edulis) [C]. St. Andrews, New Brunswick, Canada: Open Ocean Aquaculture IV Symposium. June 17-20, 2001: 75-76.

[3]Wang Jing-kun, LIU Zhen-chang, YANG Hong-sheng. Virtual design and simulation research for the float raft of deep-sea farming[J]. Fishery Modernization, 2008, 35(1):32-35.

[4]Li Guan-Yin, Huang Cai-Cheng. Dynamic analysis of deepwater floating raft oyster rack[C]. The 31th Marine Engineering Conference. Taiwan: National Sun Yat-Sen University. 2009:553-560.

[5]Deng Tui. Numerical simulation of raft-cultivation model under wave action [D]. Dalian University of technology. 2010.

[6]Deng Tui, Dong Guo-Hai, Zhao Yun-Peng, Li Yu-Cheng. Numerical simulation of raft-cultivation model under wave action[J]. Fishery Modernization, 2010,37(2):26-30.

[7]Cui Yong, Jiang Zeng-Jie, Guan Chang-Tao, Wan Rong. Numerical simulation of dynamic response of long-line culture facility to the current[J]. Progress In Fishery Sciences. 2012, 33(3):102-107.

[8]Li Yu-Chent, Teng Bin. Wave Action on Maritime Structures [M]. Ocen Press, 1992.

[9]Zhang Guang-Fa. 3D Numeric Simulation for Launching Jacket [D]. Dalian University of Technology, 2007.

[10]Shao Li-Min. The formation and level of sea wave [C]. The conference of new technology and new experience on preventing the ship accident(Book B). 2004.

[11]Cong Yan-Rong. Wave mechanics for Ocean Engineering[M]. Tianjin University Press, 1991. 